\title{
Kleine Änderung bei den Krankenfahrten
}

Krankenfahrten können nur in bestimmten Ausnahmefällen zulasten der GKV verordnet werden, damit diese die Kosten übernimmt. Dazu gehören momentan Transporte zur stationären Behandlung - auch in Notfällen - sowie Fahrten zu stationsersetzenden Operationen ( $\$ 115$ b SGB V) und zur vor- und nachstationären Behandlung im Krankenhaus ( $\$ 115 \mathrm{a}$ SGB V). Etwas komplizierter wird es bei Fahrten zu Dialysebehandlungen, onkologischer Strahlentherapie und onkologischer Chemotherapie oder für Patienten, die einen Schwerbehindertenausweis mit Merkzeichen „aG“, „BI“ oder „H“ oder einen Einstufungsbescheid in die Pflegestufe 2 oder 3 vorlegen. Diese Fahrten sind ebenfalls verordnungsfähig und werden in der Regel auch von der Kasse bezahlt, es bedarf aber vorab einer Genehmigung der Kasse. In allen anderen Fällen ist eine Verordnung ausgeschlossen. Allenfalls kann sich der Patient im Erstattungsverfahren an seine Kasse wenden.

\section{MMW-KOMMENTAR}

Eine Anpassung gibt es jetzt beim Punkt "onkologische Chemotherapie". Die neue Formulierung lautet „parenterale antineoplastische Arzneimitteltherapie/parenterale onkologische Chemotherapie". Hintergrund dieser Anpassung ist, dass der bisherige Begriff nicht das heute viel breitere Behandlungsspektrum abdeckt. In der Onkologie werden zunehmend antineoplastische Arzneimitteltherapien eingesetzt, die keine klassische Chemotherapie darstellen, sondern anderen Wirkprinzipien folgen - man denke z. B. an monoklonale Antikörper. Viele dieser neuen
Therapien erfolgen aber ebenfalls in einer vergleichbar hohen Behandlungsfrequenz über einen längeren Behandlungszeitraum. Dabei beeinträchtigen diese Therapien oder die zugrunde liegende Krankheit - den Patienten ebenfalls in einer Weise, dass eine Beförderung zur Schadensvermeidung unerlässlich ist.

Es ist wichtig, diese durchaus verwirrenden Regelungen zu beachten und ggf. auf Nummer sicher zu gehen. Mit dem im Juli 2015 verabschiedeten GKV-Versorgungsstärkungsgesetz wurde nämlich festgelegt, dass die Wirtschaftlichkeit der Versorgung mit ärztlich verordneten Leistungen ab 2017 anhand von Vereinbarungen zwischen KVen und Krankenkassen auf der Landesebene geprüft werden kann. Diese Prüfung kann auch Verordnungen für Krankentransporte umfassen - und einen Regress nach sich ziehen.

\section{Schließt Nr. 01435 alle anderen Leistungen aus?}

- Die Nr. 01435 EBM steht für die sogenannte Haus-/Fachärztliche Bereitschaftspauschale und hat keine Zeitvorgabe. Das bedeutet, sie geht nicht in die Wirtschaftlichkeitsprüfung nach Zeitvorgaben ein. Sie kann einmalig im Quartal für eine telefonische Beratung des Patienten im Zusammenhang mit einer Erkrankung berechnet werden, wenn der Patient selbst den Kontakt aufnimmt. Anstelle des Telefonats kann auch ein anderer mittelbarer Arzt-Patienten-Kontakt gemäß 4.3.1 der Allgemeinen Bestimmungen stehen, z. B. ein Gespräch mit Angehörigen oder Pflegepersonal. Die Nr. ist mit 9,18 Euro bewertet und unterliegt der Budgetierung im Regelleistungsvolumen. Bei Patienten bis zum vollendeten 12. Lebensjahr ist eine bis zu zweimalige Berechnung im Behandlungsfall möglich.

\section{MMW-KOMMENTAR}

Immer wieder taucht die Behauptung auf, dass neben der Nr. 01435 im gesamten Behandlungsfall, also im gesamten gleichen Quartal, keine anderen berechnungsfähigen EBM-Leistungen zur Abrechnung kommen können. Selbst von den Prüfgremien der KVen bekommen Ärzte das bisweilen zu hören-aber es ist eindeutig falsch! Vielmehr ist es so, dass die Nr. 01435 nicht berechnet werden kann, wenn im selben Quartal eine Versicherten-, Grund- und/oder Konsiliarpauschale zur Abrechnung kommt. Das ist ein Unterschied!

Im Übrigen ist die Regel auf den Arztfall bezogen, nicht auf den Behandlungsfall. Das hat konkrete Auswirkungen auf die Betreuung in Gemeinschaftspraxen. Wenn hier einer der Praxispartner mit seiner lebenslangen Arztnummer (LANR) bei einem Patienten die Versichertenpauschale berechnet, kann ein anderer Partner durchaus die Nr. 01435 einmalig im selben Quartal berechnen schließlich kennzeichnet er sie mit einer anderen LANR. Man muss nur beachten, dass dann an diesem Tag außer Kostenpauschalen keine anderen Leistungen von diesem Praxispartner beim gleichen Patienten zum Ansatz kommen können. Das geht dann aber wieder an anderen Tagen des Quartals.

Nr. 01435 EBM: Eine Telefonberatung nach Kontaktaufnahme durch den Patienten. 